Review

\title{
Ultrasound of the elbow with emphasis on detailed assessment of ligaments, tendons, and nerves
}

\author{
Michel De Maeseneer ${ }^{\mathrm{a}, *}$, Monica Kalume Brigido ${ }^{\mathrm{b}}$, Marijana Antic ${ }^{\mathrm{a}}$, Leon Lenchik ${ }^{\mathrm{c}}$, \\ Annemieke Milants ${ }^{\mathrm{a}}$, Evie Vereecke ${ }^{\mathrm{d}}$, Tjeerd Jager ${ }^{\mathrm{e}}$, Maryam Shahabpour ${ }^{\mathrm{a}}$ \\ a Department of Radiology, Universitair Ziekenhuis Brussel, Brussels, Belgium \\ b Department of Radiology, University of Michigan, Ann Arbor, MI, USA \\ ${ }^{c}$ Department of Radiology, Wake Forest University, Winston-Salem, NC, USA \\ d Department of Anatomy, KULAK, Katholieke Universiteit Leuven, Campus Kortrijk, Kortrijk, Belgium \\ e Aalsters Stedelijk Ziekenhuis, Aalst, Belgium
}

\section{A R T I C L E I N F O}

\section{Article history:}

Received 4 September 2014

Received in revised form 5 December 2014

Accepted 9 December 2014

\section{Keywords:}

Elbow-ultrasound

Elbow-anatomy

Elbow-nerves

\begin{abstract}
A B S T R A C T
The high resolution and dynamic capability of ultrasound make it an excellent tool for assessment of superficial structures. The ligaments, tendons, and nerves about the elbow can be fully evaluated with ultrasound. The medial collateral ligament consists of an anterior and posterior band that can easily be identified. The lateral ligament complex consists of the radial collateral ligament, ulnar insertion of the annular ligament, and lateral ulnar collateral ligament, easily identified with specialized probe positioning. The lateral ulnar collateral ligament can best be seen in the cobra position. On ultrasound medial elbow tendons can be followed nearly up to their common insertion. The pronator teres, flexor carpi radialis, palmaris longus, and flexor digitorum superficialis can be identified. The laterally located brachioradialis and extensor carpi radialis longus insert on the supracondylar ridge. The other lateral tendons can be followed up to their common insertion on the lateral epicondyle. The extensor digitorum, extensor carpi radialis brevis, extensor digiti minimi, and extensor carpi ulnaris can be differentiated. The distal biceps tendon is commonly bifid. For a complete assessment of the distal biceps tendon specialized views are necessary. These include an anterior axial approach, medial and lateral approach, and cobra position. In the cubital tunnel the ulnar nerve is covered by the ligament of Osborne. Slightly more distally the ulnar nerve courses between the two heads of the flexor carpi ulnaris. An accessory muscle, the anconeus epitrochlearis can cover the ulnar nerve at the cubital tunnel, and is easily identified on ultrasound. The radial nerve divides in a superficial sensory branch and a deep motor branch. The motor branch, the posterior interosseous nerve, courses under the arcade of Frohse where it enters the supinator muscle. At the level of the dorsal wrist the posterior interosseous nerve is located at the deep aspect of the extensor tendons. The median nerve may be compressed at various sites, including the lacertus fibrosis, between the pronator teres heads, and the sublimis bridge. These compression sites can be identified with ultrasound.
\end{abstract}

CC 2014 Elsevier Ireland Ltd. All rights reserved.

\section{Introduction}

Ultrasound is often used to assess soft tissue structures about joints [1]. Ultrasound offers some advantages over MR, owing to its higher resolution. This allows better depiction of fine nerve

\footnotetext{
* Corresponding author at: Department of Radiology, Universitair Ziekenhuis Brussel, Laarbeeklaan 101, 1090 Brussels, Belgium. Tel.: +32 0491250001.

E-mail addresses: Michel.demaeseneer@uzbrussel.be (M. De Maeseneer), Mbrigido@med.umich.edu (M.K. Brigido), Misscroa@gmail.com (M. Antic), Llenchik@wakehealth.edu (L. Lenchik), Annemieke.Milants@gmail.com (A. Milants),Evie.Vereecke@kuleuven-kulak.be (E. Vereecke), Maryam.Shahabpour@uzbrussel.be (M. Shahabpour).
}

branches and better appreciation of subtle alterations in the fibrillar echotexture of tendons and ligaments. Because ultrasound is dynamic, the integrity and continuity of tendons and ligaments can be assessed in real time in positions of stress. Comparison with the asymptomatic side is possible and may help diagnose subtle abnormalities. Finally power Doppler is valuable in diagnosing tendinopathies such as tennis or golfers elbow. A disadvantage of ultrasound is that it is operator dependent and may be technically difficult. This disadvantage can be overcome by proper training.

In this work we discuss the detailed ultrasound anatomy of tendons, ligaments and nerves about the elbow, using cadaveric correlation. We include tips and tricks for evaluating difficult anatomic structures. 


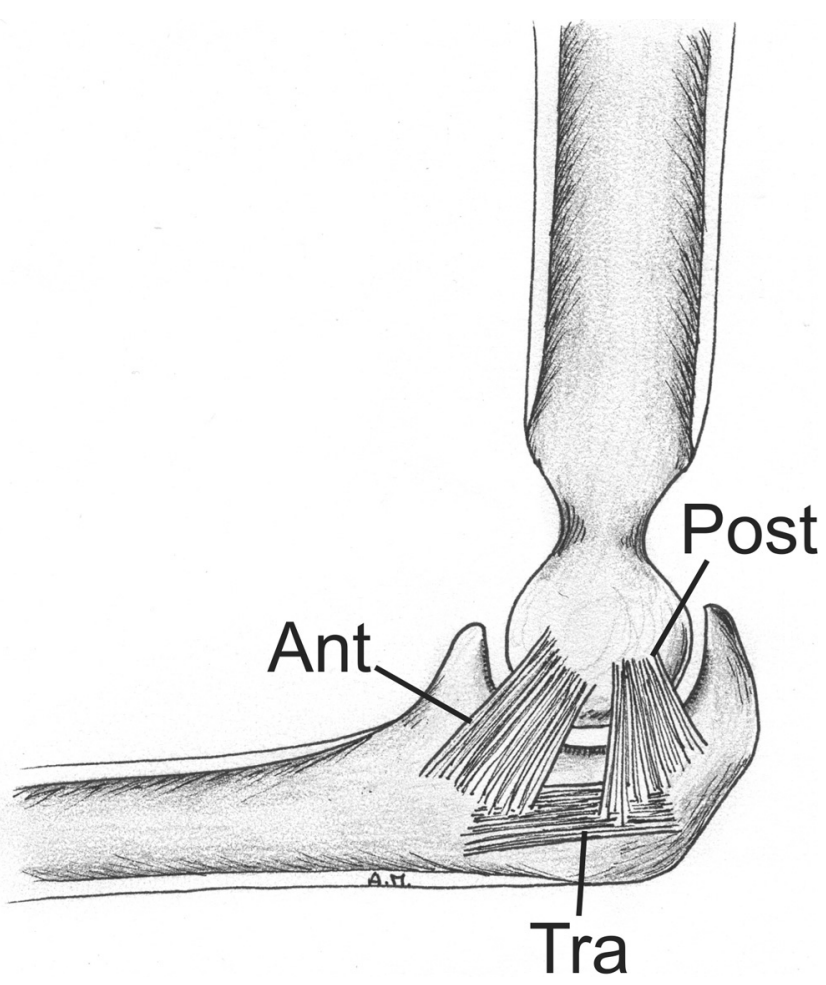

Fig. 1. Drawing of medial elbow ligaments. Note anterior (Ant), posterior (Post) and transverse (Tra) components of the ulnar collateral ligament.

\section{Discussion}

\subsection{Medial ligaments}

The ulnar collateral ligament (UCL) forms the major restraint to valgus stress $[1,2]$. It is commonly injured in the setting of throwing injuries and sports. The UCL consists of an anterior, posterior and transverse band (Fig. 1). The anterior band is mechanically the most important. To image the anterior band the elbow should be put in slight flexion as this allows optimal alignment of the ultrasound probe along the course of the ligament. An excellent trick is to hook the 2 nd to 4 th fingers of the operator behind the medial epicondyle from an anterior approach and let the probe slide onto the fingers from anteriorly. The probe is then positioned along the course of the ligament (Fig. 2).

On MR arthrography the distal attachment of the UCL to the ulna should be tightly attached to the sublime tubercle without contrast interposition between the ligament and ulna. On ultrasound, however, the ligament may appear to insert more distally.

In some patients, the proximal insertion of the anterior band may be somewhat broader or multifibrillar and this should not be taken for a sign of abnormality. It may often be difficult on one static image to separate the ligament fibers from the adjacent fatty tissue. Slightly tilting the probe back and forth uses anisotropy to better differentiate the ligament from adjacent fat (Fig. 2). Along the posteromedial aspect of the elbow the posterior band of the UCL can be seen. Probe positioning is similar to that for assessment of the ulnar nerve and the ligament of Osborne (Fig. 3). The posterior band of the UCL spans the humeroulnar joint like a hammock and forms the bottom of the cubital tunnel. The ulnar nerve is located adjacent to it.

The transverse component of the UCL courses between the ulnar insertions of the anterior and posterior bands, and is less important.

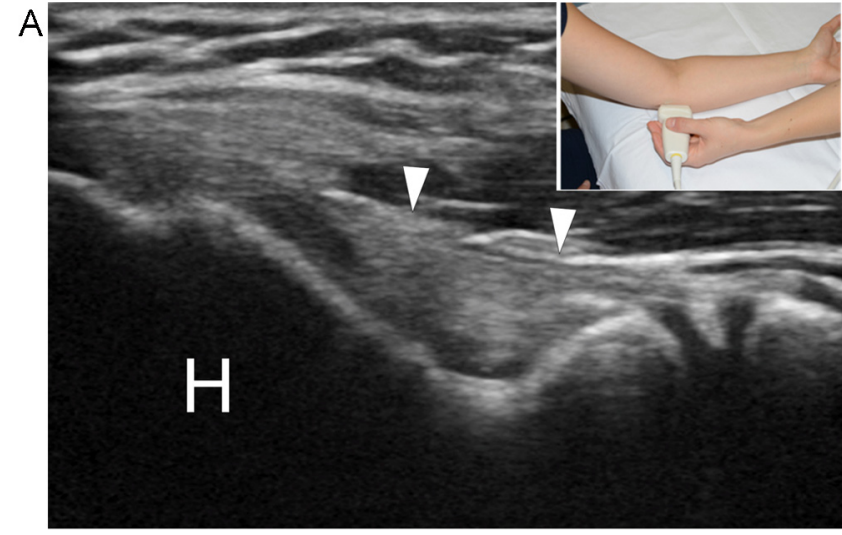

$\mathrm{B}$

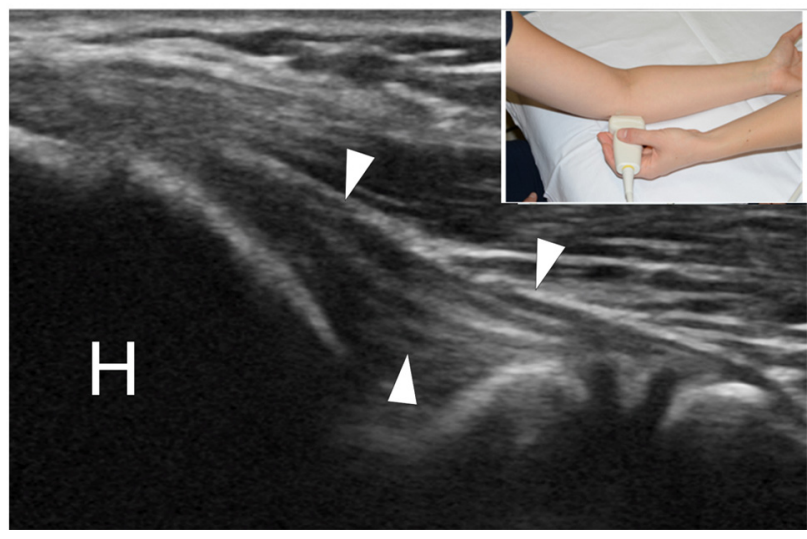

Fig. 2. (A, B) Longitudinal view of anterior component of the ulnar collateral ligament (arrowheads). Note that with tilting of the probe anisotropy can be used to better outline the ligament. In (A) ligament is difficult to differentiate from adjacent fat. Better differentiation is seen in (B). Also note multifibrillar aspect. $\mathrm{H}$, humerus Insert, probe position.

\subsection{Lateral ligaments}

The anatomy of the lateral ligaments is complex (Fig. 4) [2]. The ligaments include the radial collateral ligament (RCL), ulnar insertion of the annular ligament, and lateral ulnar collateral ligament (LUCL). Probe placement for the RCL is similar to that for assessment of the extensor tendons of the elbow in the longitudinal plane (Fig. 5). The concavity in the distal aspect of the humerus is identified for correct probe placement. Various tubercles may be identified on the lateral epicondyle. The radial collateral ligament

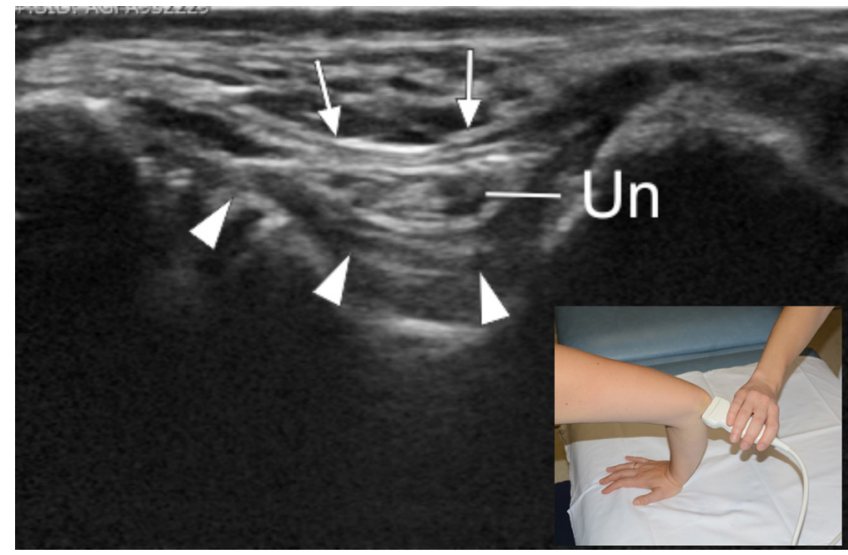

Fig. 3. Longitudinal view of posterior component of the ulnar collateral ligament Note ligament (arrowheads) that forms a hammock for the ulnar nerve (Un). Ulnar nerve is covered by Osborne's ligament (arrows). Insert, probe positioning. 


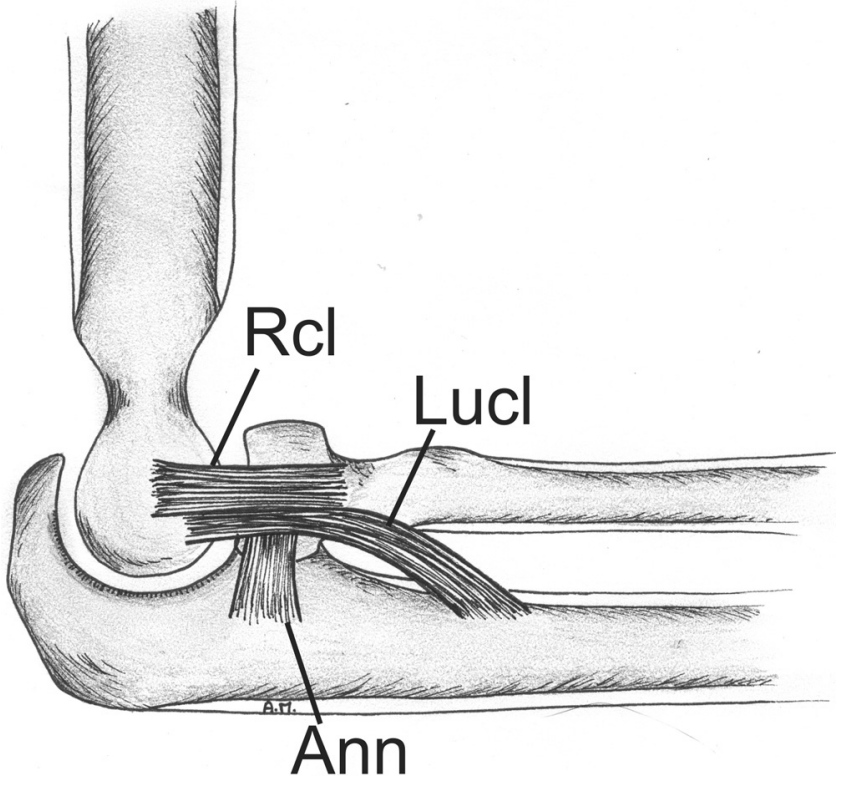

Fig. 4. Drawing of lateral elbow ligaments. Note radial collateral ligament (Rcl), ulnar insertion of the annular ligament (Ann), and lateral ulnar collateral ligament (Lucl).

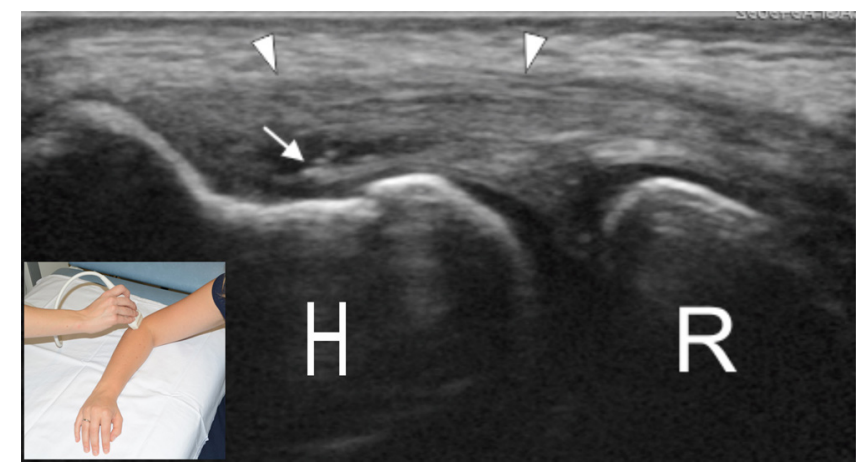

Fig. 5. Longitudinal view of the radial collateral ligament. In this patient a tear was present at the deep aspect of the extensor carpi radialis brevis tendon (arrowheads), outlining the radial collateral ligament (arrow). $\mathrm{H}$, humerus; $\mathrm{R}$, radius. Insert, probe positioning.

inserts on the humeral epicondyle in the groove between the anterior and posterior tubercle. From here it courses toward the radial head where it joins the annular ligament. Occasionally it can be separated from the overlying extensor tendons by a delicate hyperechoic line. In the transverse plane anisotropy helps to differentiate the ligament from the overlying tendon (Fig. 6).

The annular ligament forms a band around the radial head [3]. The annular ligament has an ulnar insertion. When the probe is placed in a transverse plane with respect to the radial head the thin fibers of the annular ligament can be seen to insert on the ulna (Fig. 7). The proximal part of the LUCL also inserts on the humerus in the same area as the RCL but somewhat more toward the radial side $[4,5]$. Typically, however, the LUCL and RCL cannot be separated along their proximal course. Distally the LUCL courses more distally than the RCL and inserts on the supinator crest of the ulna. In this insertion area the ligament typically fans out. To visualize the LUCL we start with the position for the annular insertion which is transverse with regard to the radial head. From this position, the probe is then slightly tilted anteriorly in between the radius and the supinator crest (Fig. 8). Typically a few delicate vascular structures are seen between the LUCL and adjacent muscles, and these vessels

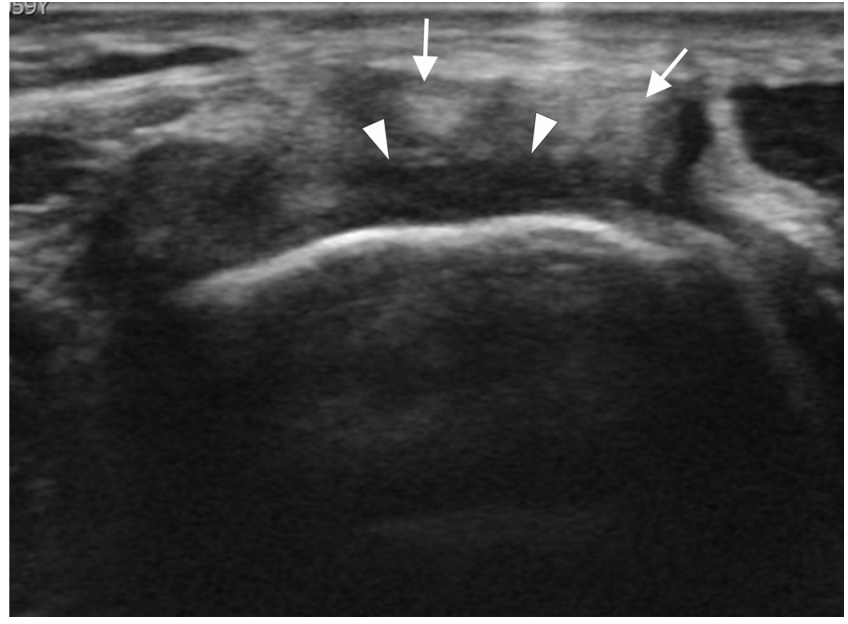

Fig. 6. Short axis view of the tendon and ligament insertion on the lateral humeral epicondyle. By using anisotropy, the radial collateral ligament (arrowheads), can be differentiated from the overlying tendon fibers (arrows).

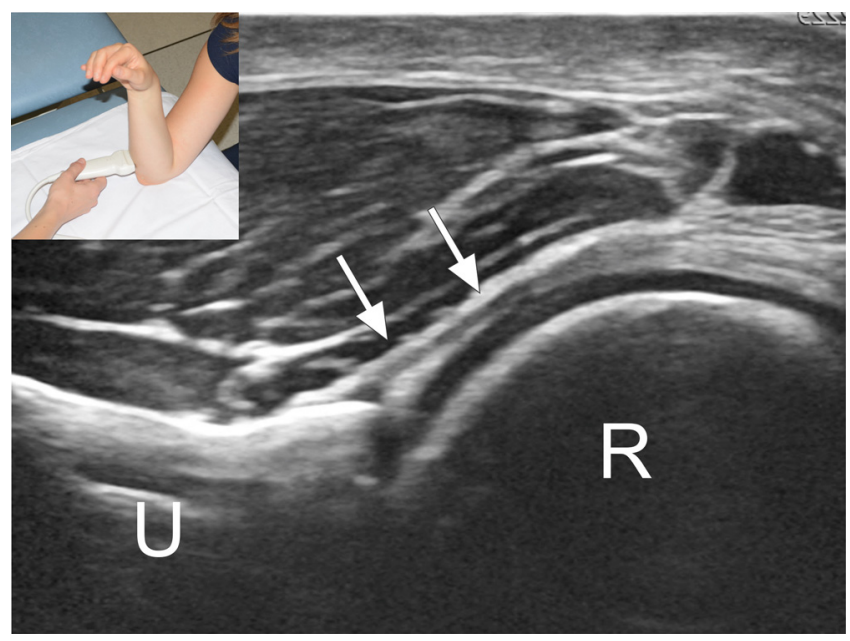

Fig. 7. Longitudinal view of the ulnar insertion of the annular band (arrows). $R$, radius; $U$, ulna. Insert, probe position.

constitute an excellent landmark. We prefer to assess the annular insertion and LUCL with the arm in the cobra position. In the cobra position the LUCL is typically much better outlined likely because it is placed under tension (Fig. 8).

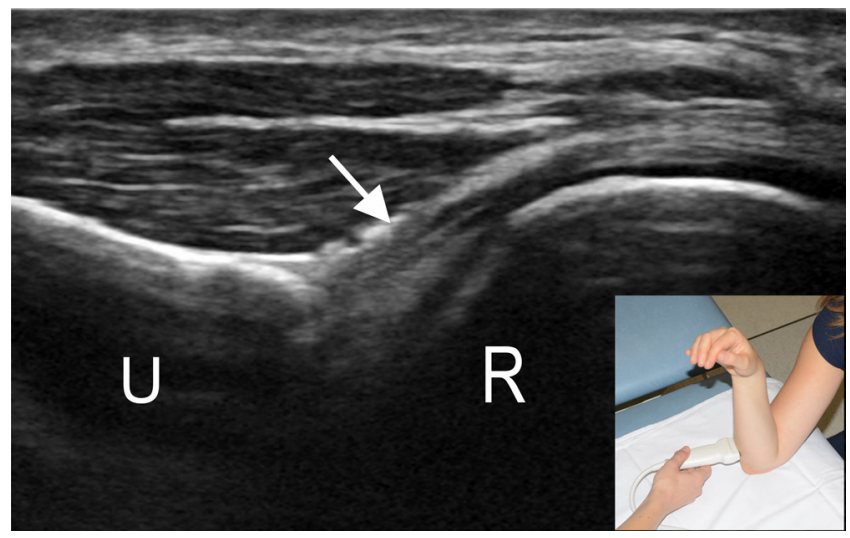

Fig. 8. Longitudinal view of the lateral ulnar collateral ligament (arrow). U, ulna; R, radius. Insert, probe position. Probe position is more tilted anteriorly compared to that used for imaging the annular insertion.

Please cite this article in press as: De Maeseneer M, et al. Ultrasound of the elbow with emphasis on detailed assessment of ligaments, tendons, and nerves. Eur J Radiol (2015), http://dx.doi.org/10.1016/j.ejrad.2014.12.007 


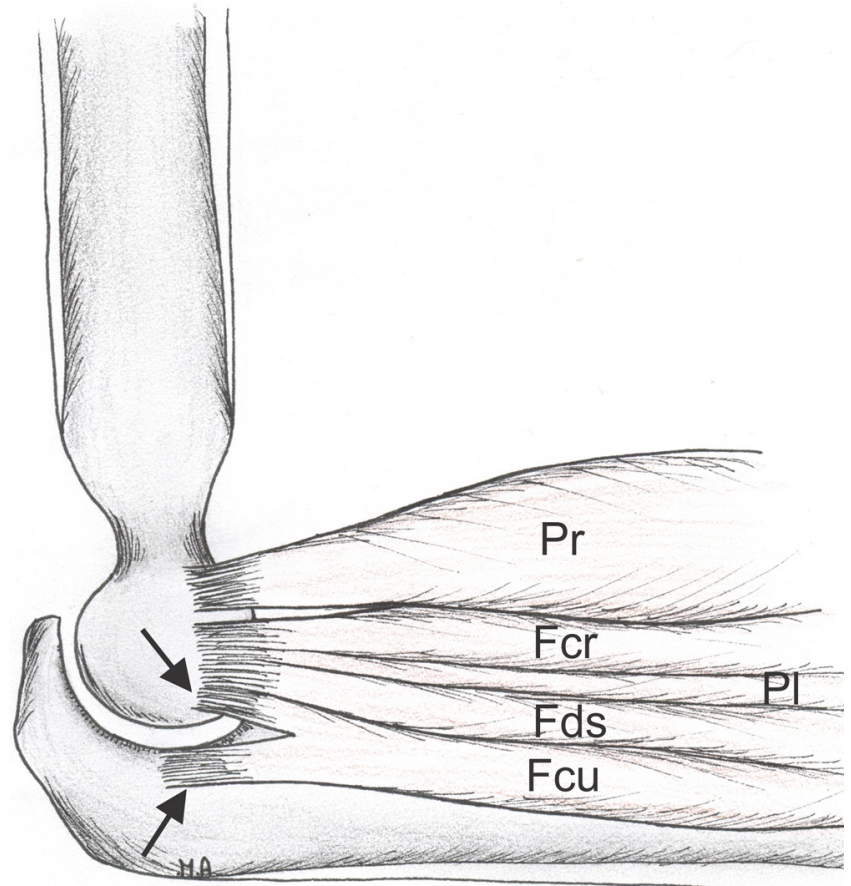

Fig. 9. Drawing of medial elbow tendons. Note pronator teres (Pr), flexor carpi radialis (Fcr), Palmaris longus (Pl), flexor digitorum superficialis (Fds), and humeral and ulnar heads (arrows) of the flexor carpi ulnaris (Fcu).

\subsection{Medial tendons}

Although the medial tendons have a common insertion on the medial epicondyle they can be followed separately very close to their insertion (Fig. 9) [1,6,7]. To visualize these different insertions we use the 'elevator' method. We start at the wrist level and identify the flexor carpi radialis tendon which is easily outlined. We follow the tendon proximally as its muscle belly forms (Fig. 10). As we follow the flexor carpi radialis muscle proximally, the pronator teres muscle becomes visible anterior to it. Posterior to the flexor

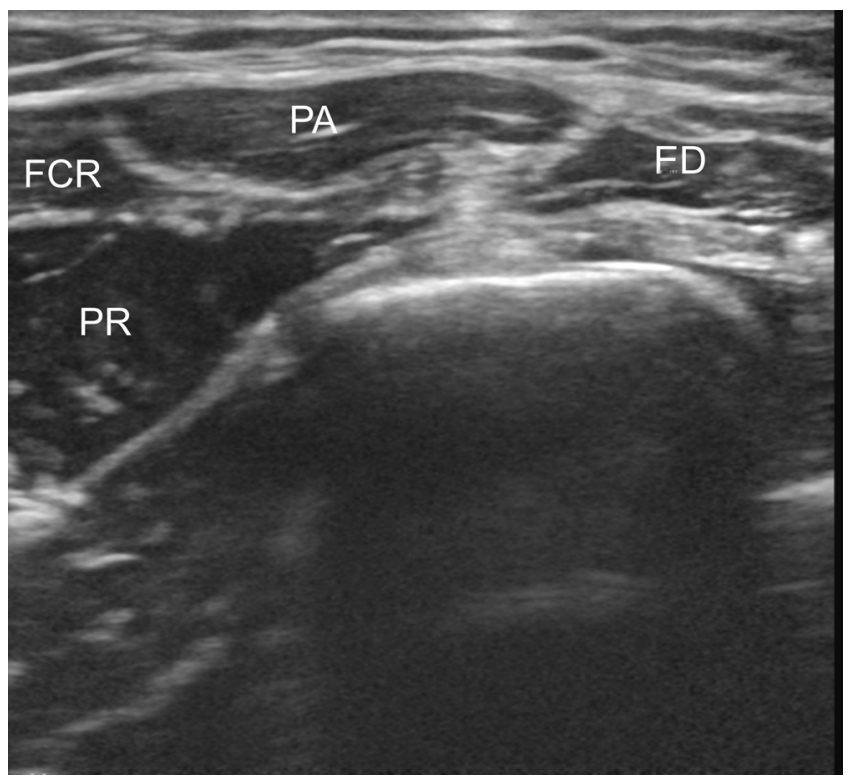

Fig. 10. Short axis view of medial aspect of elbow just before insertion of the flexor tendons on the epicondyle. From anterior to posterior, note pronator teres (PR), flexor carpi radialis (Fcr), Palmaris longus (PA), and flexor digitorum superficialis (Fds).

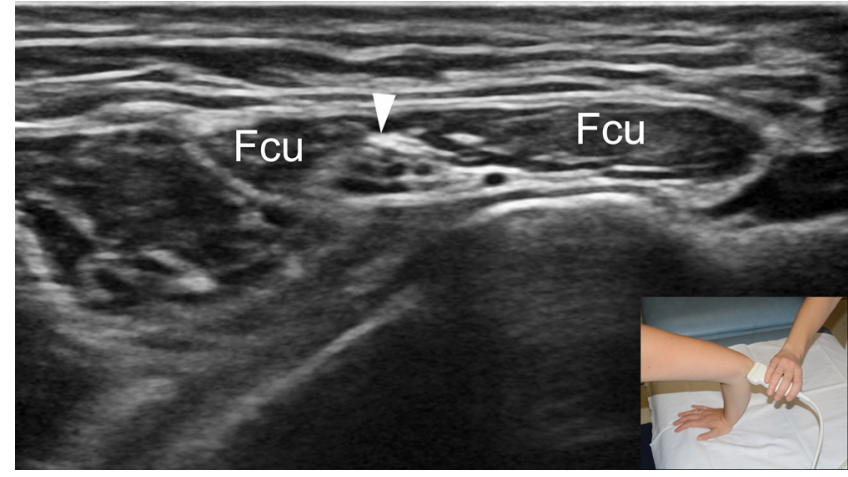

Fig. 11. More posterior relative to Fig. 10. Note ulnar nerve (arrowhead) between both heads of the flexor carpi ulnaris (Fcu).

carpi radialis muscle the flexor digitorum superficialis muscle is seen. When present, a small muscle belly and tendon can be seen in between the flexor carpi radialis and flexor digitorum superficialis muscle. This structure corresponds to the palmaris longus muscle and tendon. We identify the flexor carpi ulnaris from a posterior approach. Just inferior to the humeral epicondyle and the ulna, the two muscle bellies form a dumbbell shaped structure covering the ulnar nerve. It is at this level that we also like to identify the ulnar nerve (Figs. 11 and 12).

\subsection{Lateral tendons}

Various bony tubercles and ridges can be seen on the lateral epicondyle and form important landmarks (Fig. 13). We first identify the extensor tendons in the longitudinal plane (Fig. 14) [1,6,8]. In this plane a concavity can be seen in the humerus where the tendons insert (Fig. 14). This approach is similar to that for the RCL. We then turn the probe in the transverse plane. Although the different tendons form a common insertion, they can be followed separately nearly up to the insertion (Fig. 15). Most anteriorly, the extensor carpi radialis brevis (ERCB) can be easily identified because its lateral edge has a prominent beak like appearance. Toward the medial side the muscle of the ECRB cannot be separated from the extensor carpi radialis longus muscle (ECRL). Superficial to the beak of the ECRB, the muscle belly of the extensor digitorum (ED) can be seen (Fig. 15). Lateral to the ED, a small muscle and tendon can be visualized corresponding to the extensor digiti minimi. Flexing and extending the little finger helps identify this muscle. Lateral to the extensor digiti minimi the extensor carpi ulnaris is seen. Along the

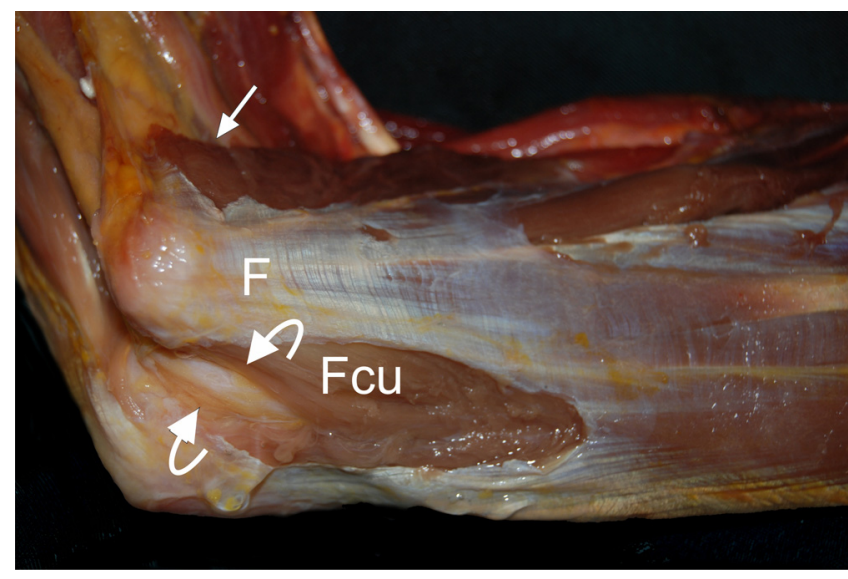

Fig. 12. Dissection of medial aspect of elbow. Note flexor tendon insertion (F) with pronator teres (arrow) located most superiorly. Note flexor carpi ulnaris (Fcu). Humeral and ulnar heads are pointed out by curved arrows. 

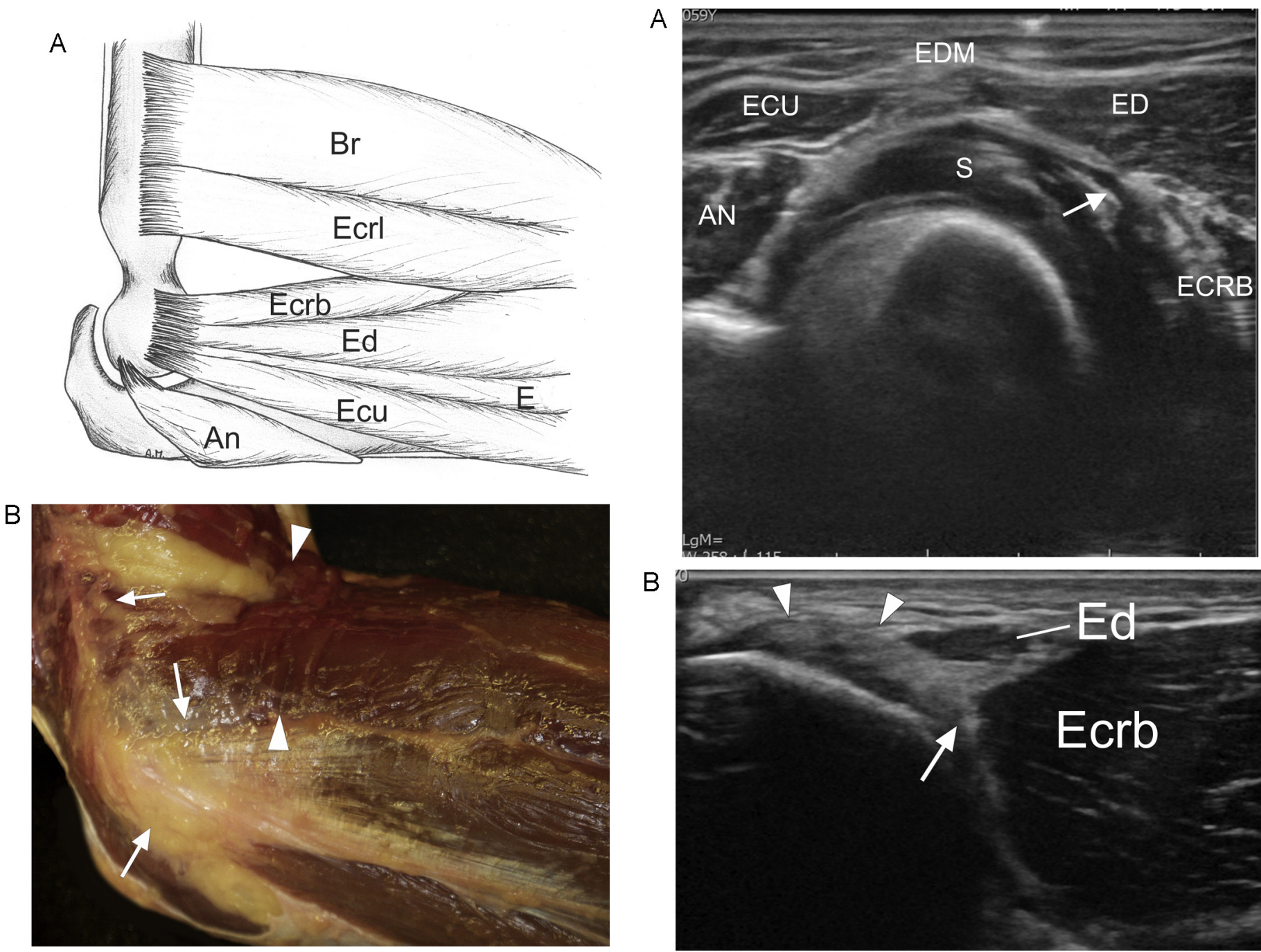

$\mathrm{B}$

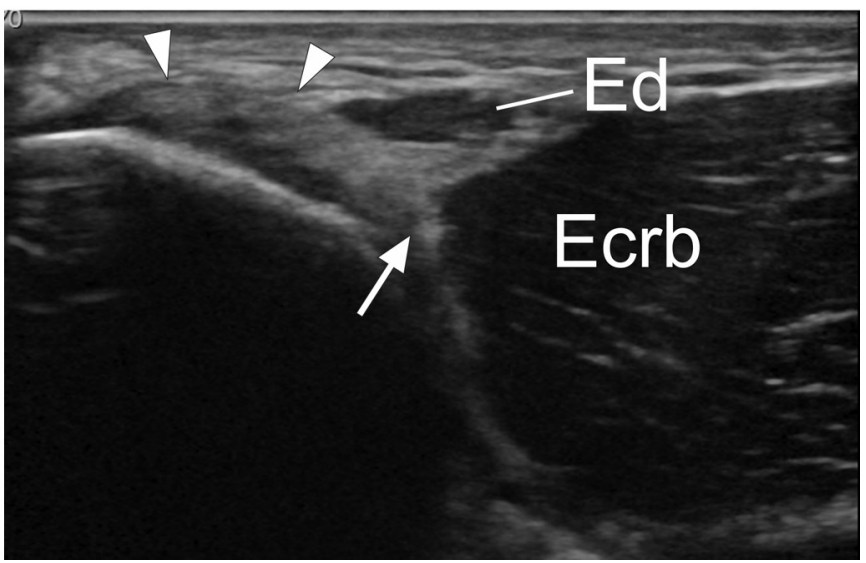

Fig. 13. (A, B) Drawing (A) and dissection (B) of the lateral aspect of the elbow. (A) Note insertion of tendons on the lateral epicondyle: extensor carpi radialis brevis (Ecrb), extensor digitorum (Ed), extensor digiti minimi (E), and extensor carpi ulnaris (Ecu). Anconeus inserts more posteriorly. Note insertion of brachioradialis $(\mathrm{Br})$ and extensor carpi radialis longus (Ecrl) on supracondylar ridge. (B) Note insertion of flexor tendons on lateral epicondyle (between arrows). Note more superior location of brachioradialis and extensor carpi radials longus (between arrowheads) inserting on supracondylar ridge (short arrow).

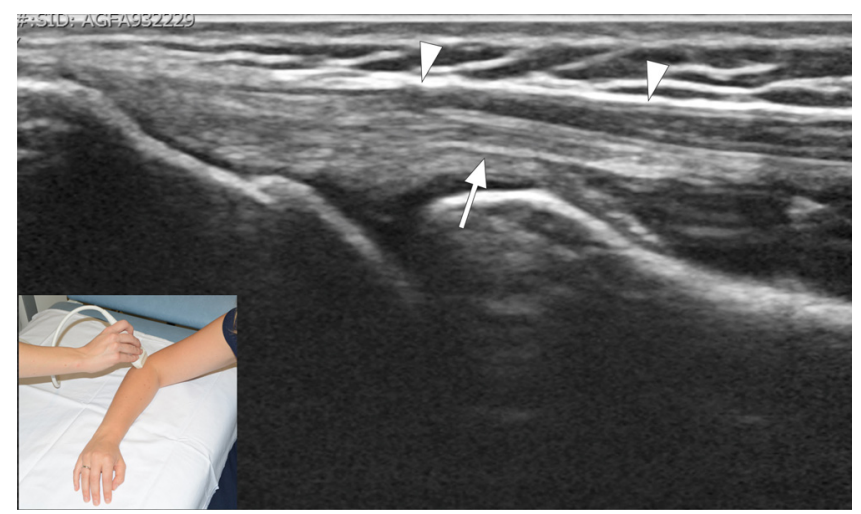

Fig. 14. Longitudinal view of lateral epicondyle. Note extensor carpi radialis brevis tendon (arrow) covered by extensor digitorum muscle (arrowheads). Insert, probe position.

Fig. 15. Short axis images along lateral epicondyle just before insertion of tendons. (A) From posterior to anterior note anconeus (AN), extensor carpi ulnaris (ECU), extensor digiti minimi (EDM), extensor digitorum (ED), and extensor carpi radialis brevis (ECRB) with its beak (arrow). (B) Detail view of slightly more proximal image compared to (A). Note 'beak' (arrow) of extensor carpi radialis brevis (Ecrb). Superficial to it extensor digitorum muscle fibers are seen (Ed). 'Beak' is oriented toward common insertion (arrowheads). S, supinator muscle.

lateral epicondyle an anterior, posterior, and superior tubercle can be identified. The insertion of the RCL is located in between the anterior and posterior tubercle. Anisotropy artifact can be used to differentiate it from the tendons (Fig. 6). Along the supracondylar ridge, the insertion of the brachioradialis and extensor carpi radialis longus are seen (Fig. 16).

\subsection{Biceps tendon}

Investigation of the biceps tendon from an anterior approach may prove quite difficult. To obtain a complete evaluation of this tendon we typically combine different approaches [9]. First, we identify the biceps tendon in the transverse plane just above the elbow joint. At this level the biceps tendon is seen adjacent to the brachial artery and median nerve (Fig. 17). This has been termed the 'BAM' sign. Distally, the biceps tendon dives into the depth but its insertion is difficult to see with this approach. This approach is useful, however, to identify a bifid biceps tendon (Figs. 18 and 19). If two tendon heads are present, the long head will insert more

Please cite this article in press as: De Maeseneer M, et al. Ultrasound of the elbow with emphasis on detailed assessment of ligaments, tendons, and nerves. Eur J Radiol (2015), http://dx.doi.org/10.1016/j.ejrad.2014.12.007 


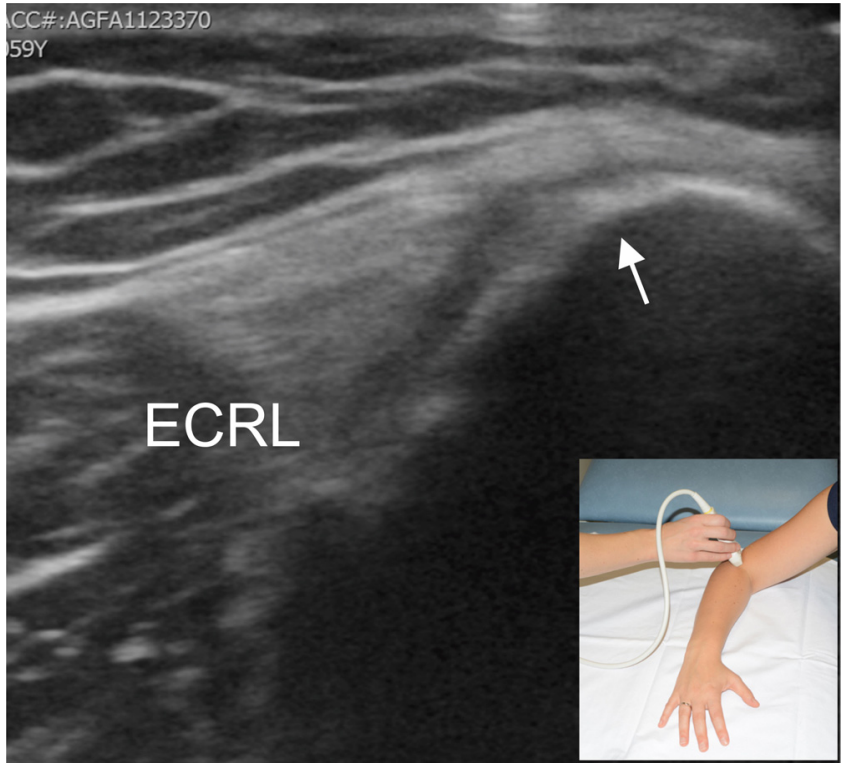

Fig. 16. Above level of epicondyle, extensor carpi radialis longus (ECRL) can be seen to insert on supracondylar ridge (arrow). Insert, probe position.

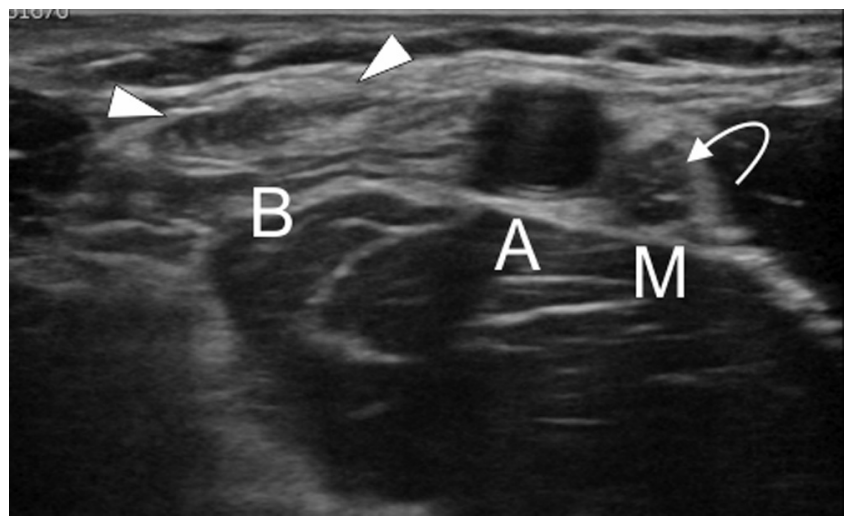

Fig. 17. Transverse section at level of elbow fold. 'BAM' relationship can be seen. Note biceps tendon (B, arrowheads), brachial artery (A), and median nerve (M, curved arrow) from lateral to medial.

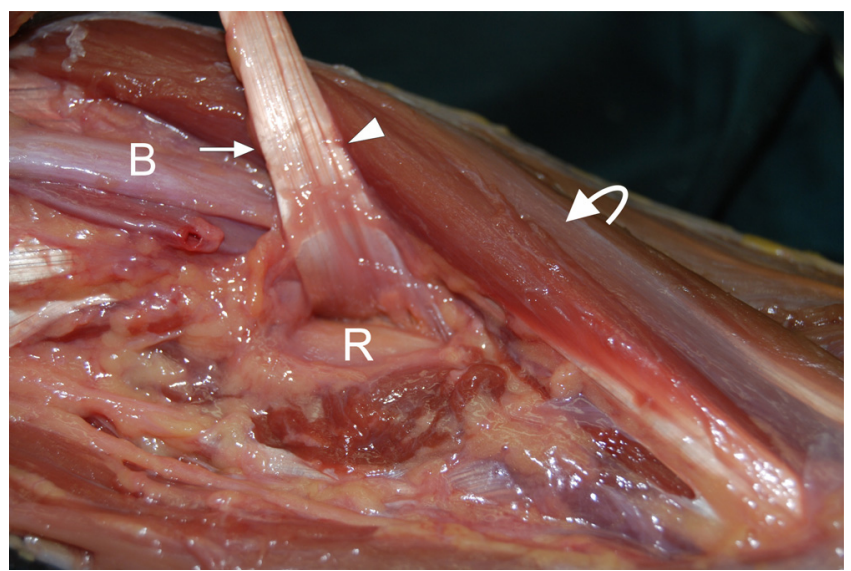

Fig. 18. Anatomical dissection show bifid appearance of distal biceps tendon. Note short head (arrowhead) more anterior relative to long head (arrow). Biceps tendon inserts on radial tuberosity (R). B, brachial artery; curved arrow, extensor muscles.

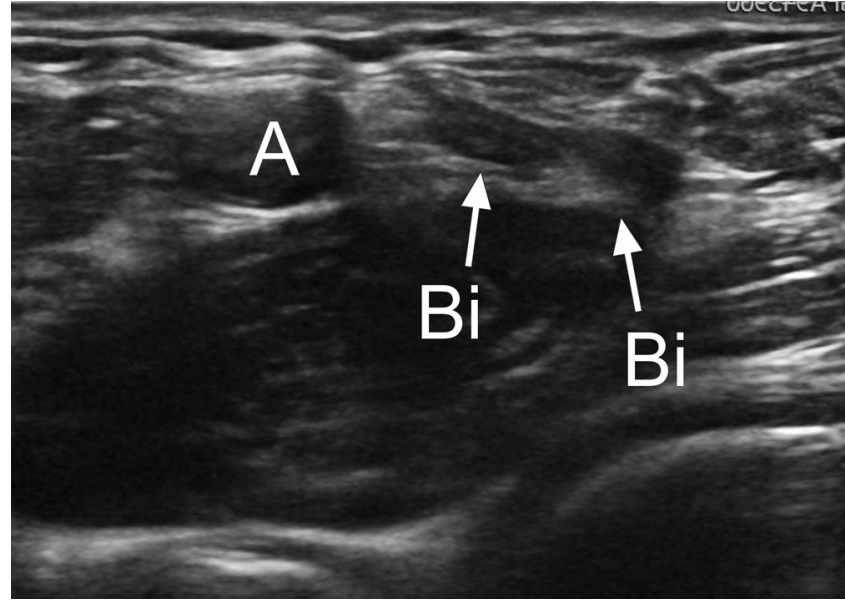

Fig. 19. Transverse anatomical section. Bifid distal biceps tendon in the transverse plane (Bi, arrows). Note artery (A).

proximally than the short head [10]. At the level where the biceps tendon is located adjacent to the brachial artery, a thin band like structure can be seen crossing over the artery which corresponds to the lacertus fibrosis (Fig. 20). The lacertus fibrosus runs from lateral to medial and fuses with the superficial flexor fascia. The basilic vein is located just superficial to the lacertus fibrosis and constitutes a good landmark. Because the vein is easily compressed, care should be taken not to exert too much pressure (Fig. 21). The

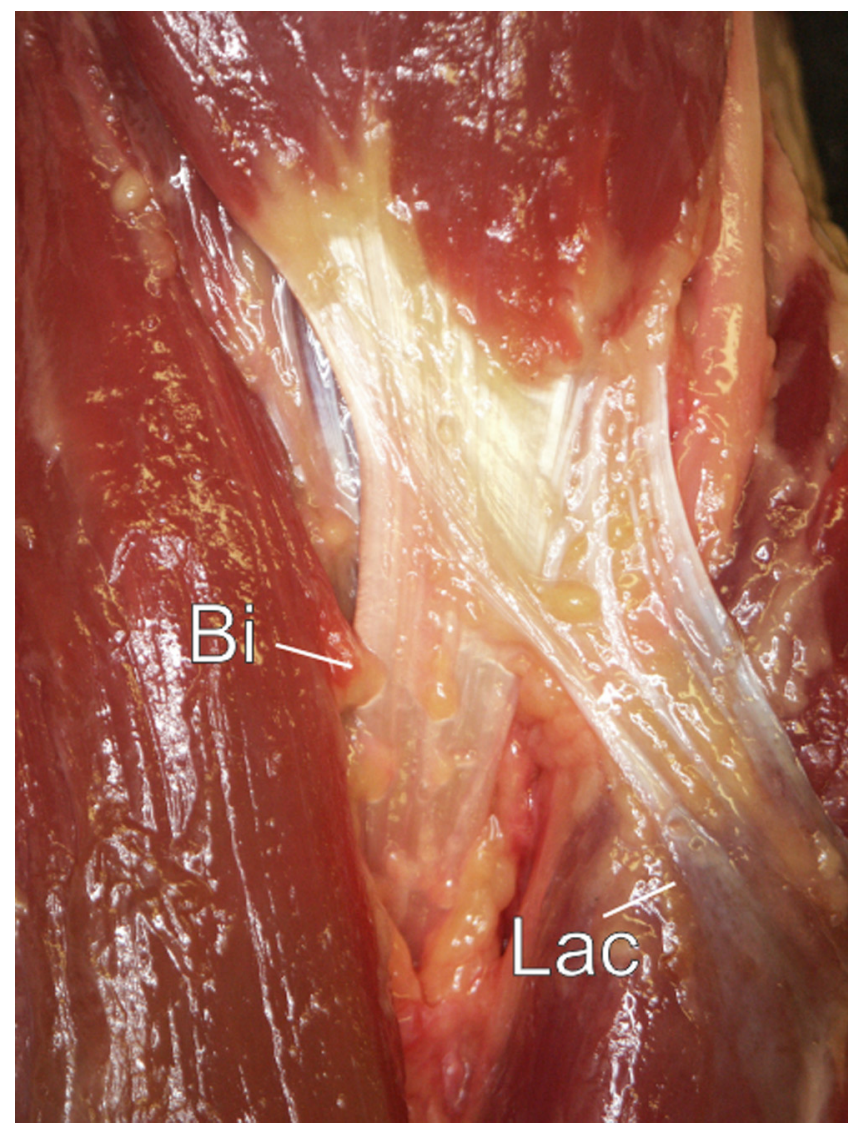

Fig. 20. Anatomical dissection. Lacertus fibrosis (Lac) can be seen to course over medial musculature from the musculotendinous junction of the biceps. Bi, biceps tendon.

Please cite this article in press as: De Maeseneer M, et al. Ultrasound of the elbow with emphasis on detailed assessment of ligaments, tendons, and nerves. Eur J Radiol (2015), http://dx.doi.org/10.1016/j.ejrad.2014.12.007 


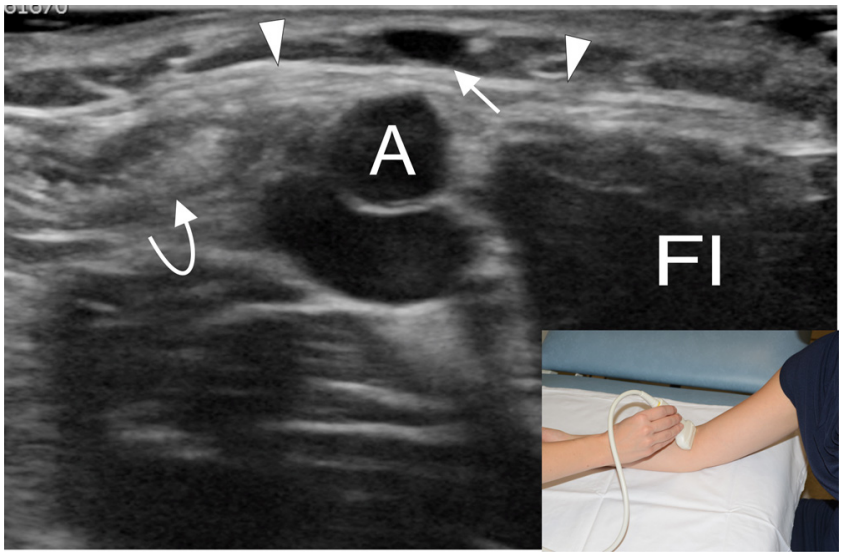

Fig. 21. Transverse ultrasound. Lacertus fibrosis can be seen as thin hyperechoic band-like structure (arrowheads) coursing over brachial artery (A). It connects to the biceps tendon (curved arrow). A good landmark is the basilica vein that is located superficial to the lacertus fibrosis (arrow). Fl, flexor tendons. Insert, probe position.

best probe position to investigate the biceps tendon consists of an oblique anteromedial approach (Fig. 22). From this position the radial tuberosity can be seen with the biceps tendon inserting on to it. The radial tuberosity is best seen with supination of the forearm. The brachial artery forms an acoustic window through which the biceps tendon can be visualized. The biceps can also be seen from a lateral approach. The probe is placed in a coronal plane along the lateral aspect of the radius. In this position, the biceps tendon can be followed deep to the supinator muscle. The insertion is difficult to see, however, as it is located behind the radius (Fig. 23). This position is useful to perform a dynamic study of the tendon. The last approach consists of a transverse approach with the forearm in the cobra position (Fig. 24). From this approach, only the insertion of the biceps tendon on the radial tuberosity is seen. Combining the

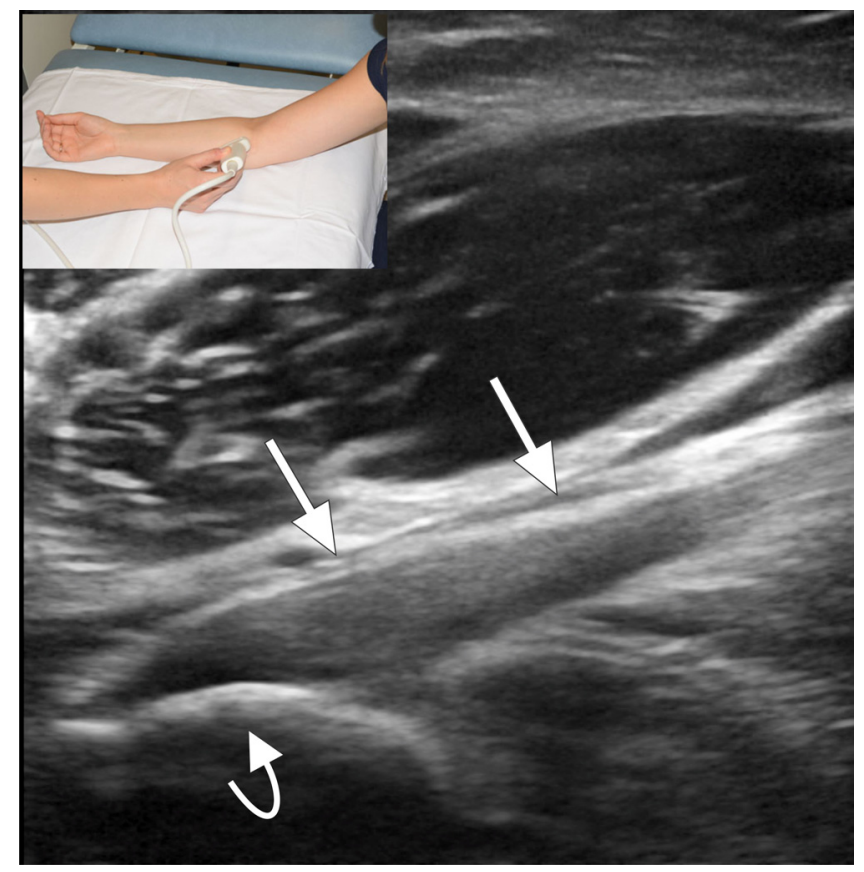

Fig. 22. Medial approach to the biceps tendon. Distal aspect of the biceps tendon (arrows) and insertion on the radial tuberosity (curved arrow) are well depicted. Insert, probe position.

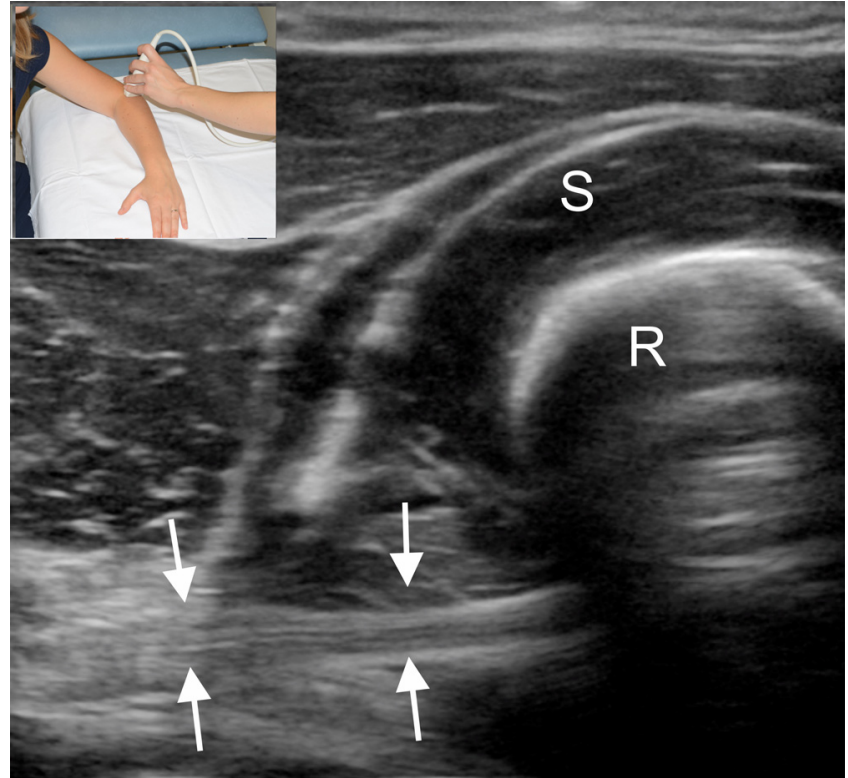

Fig. 23. Lateral approach to the biceps tendon. Biceps tendon can be seen (between arrows). Distal part is located deep to supinator muscle (S). Insertion is located behind the radius $(R)$ and cannot be seen in this position. Insert, probe position.

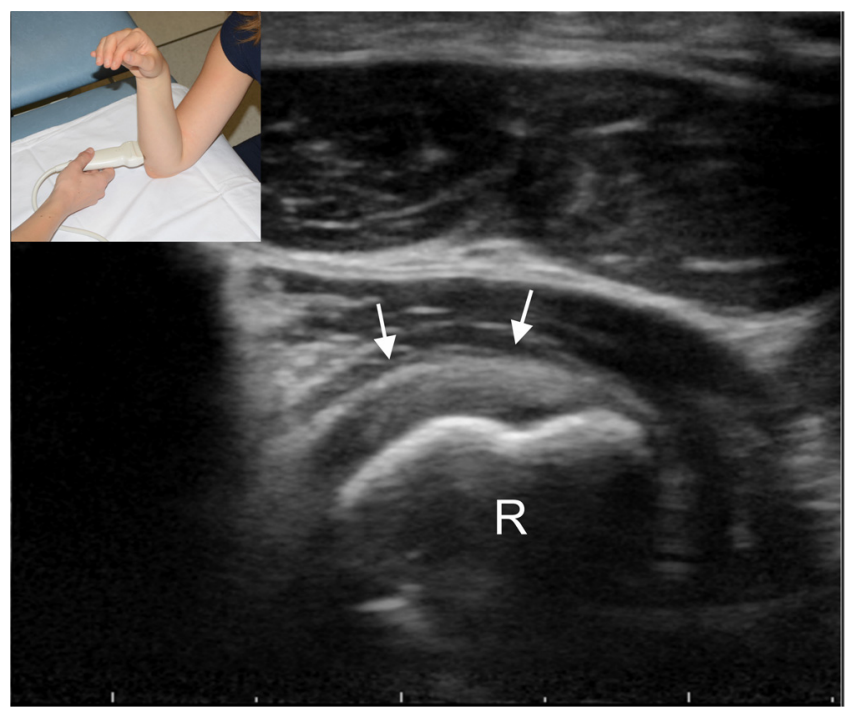

Fig. 24. Cobra position approach. With this approach only the insertion of the biceps on the radial tuberosity can be seen (arrows). R, radial tuberosity.

different approaches allows a complete examination of the distal biceps tendon.

\subsection{Ulnar nerve}

The ulnar nerve is located in the cubital tunnel, where it is covered by Osborne's ligament (Figs. 25 and 26) [11-13]. The bottom of the cubital tunnel is formed by the posterior band of the ulnar collateral ligament (Fig. 3). The ulnar nerve may be compressed between the humerus and the ligament of Osborne. With a dynamic flexion maneuver, the nerve may sublux but this also may occur in patients without neuropathy. In some patients subluxation represents a significant finding, in particular when nerve thickening is also present. In snapping triceps syndrome not only the ulnar

Please cite this article in press as: De Maeseneer M, et al. Ultrasound of the elbow with emphasis on detailed assessment of ligaments, tendons, and nerves. Eur J Radiol (2015), http://dx.doi.org/10.1016/j.ejrad.2014.12.007 


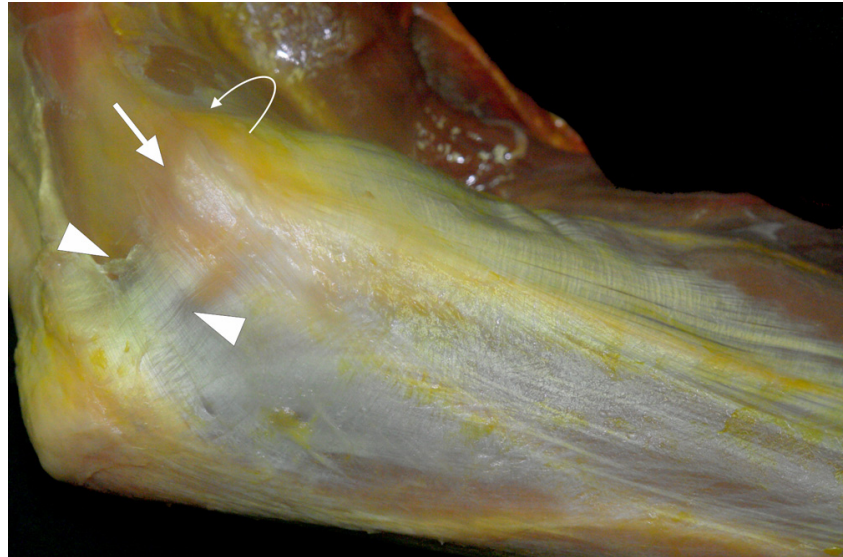

Fig. 25. Dissection of medial side of elbow. Osborne's ligament (arrowheads) can be seen covering the cubital tunnel. Note humeral epicondyle (arrow), and flexor tendon insertion (curved arrow).

nerve subluxes but also the medial head of the triceps muscle. This finding is important to report since the snapping triceps must also be addressed surgically. Instead of the ligament of Osborne, the anconeus epitrochlearis may be present (Fig. 27). This muscle may cause compression of the nerve although it is not uncommon to observe it in the asymptomatic population. Hence it needs to be correlated with focal nerve thickening.

Distal to the cubital tunnel the ulnar nerve courses between the humeral and ulnar heads of the flexor carpi ulnaris (Fig. 26). A

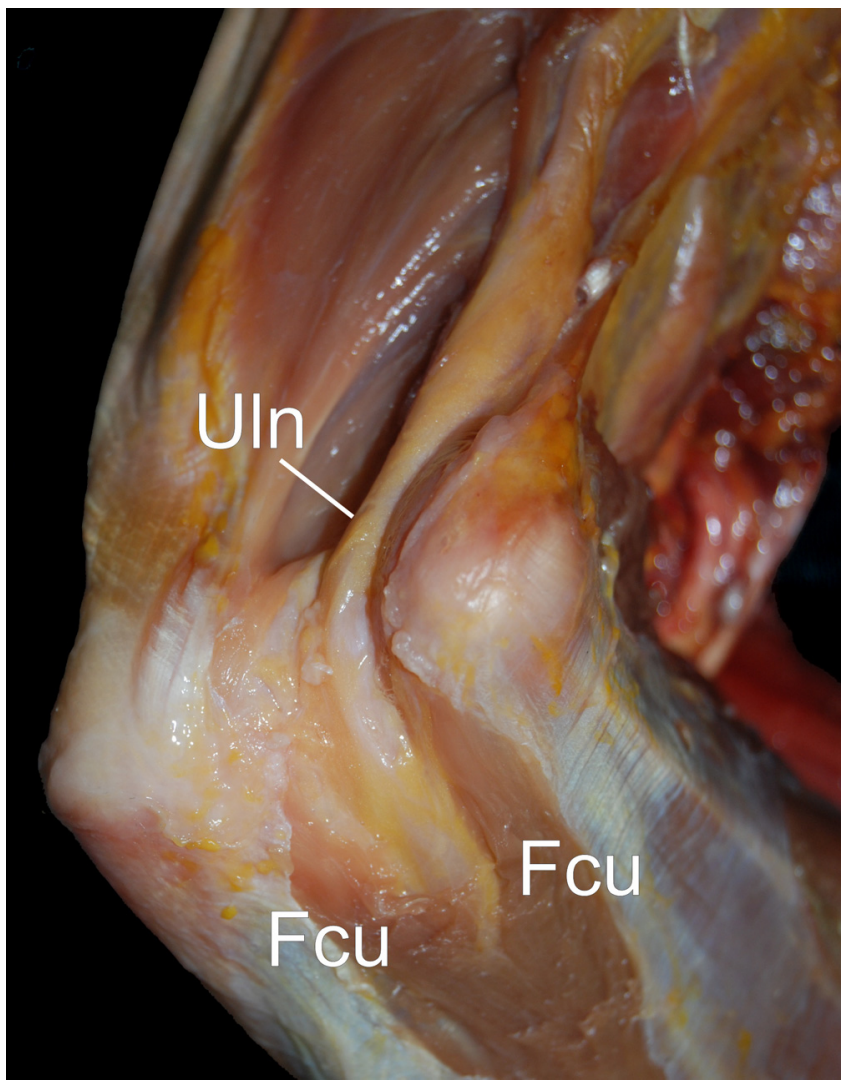

Fig. 26. Dissection. After removal of Osborne's ligament the ulnar nerve is well depicted (Uln). Distally it courses between the two heads of the flexor carpi ulnaris (Fcu).

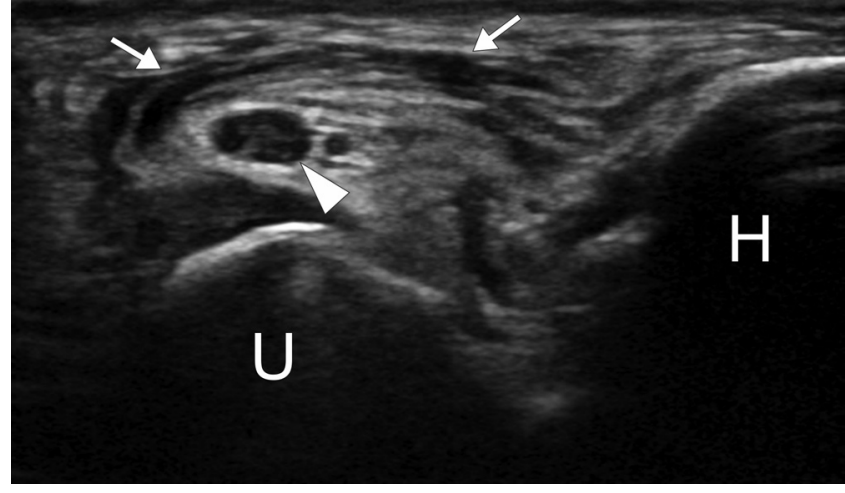

Fig. 27. Muscle variation consisting of anconeus epitrochlearis (arrows) is seen to cover ulnar nerve (arrowhead). $\mathrm{H}$, humerus; U, ulna.

fibrous arch connects both heads of the flexor carpi ulnaris and is designated the arcuate ligament. The arcuate ligament also forms a potential compression area for the ulnar nerve.

The thickness of the ulnar nerve should be thoroughly evaluated. Although standard values are less well established than for the median nerve, a value of $8 \mathrm{~mm}^{2}$ is usually employed. Focal thickening, a notch sign, and hypervascularity also are important findings [11]. It is not rare for the nerve to be bifid or trifid and in such case the different parts can be measured separately and the sum can be used for assessment of thickness.

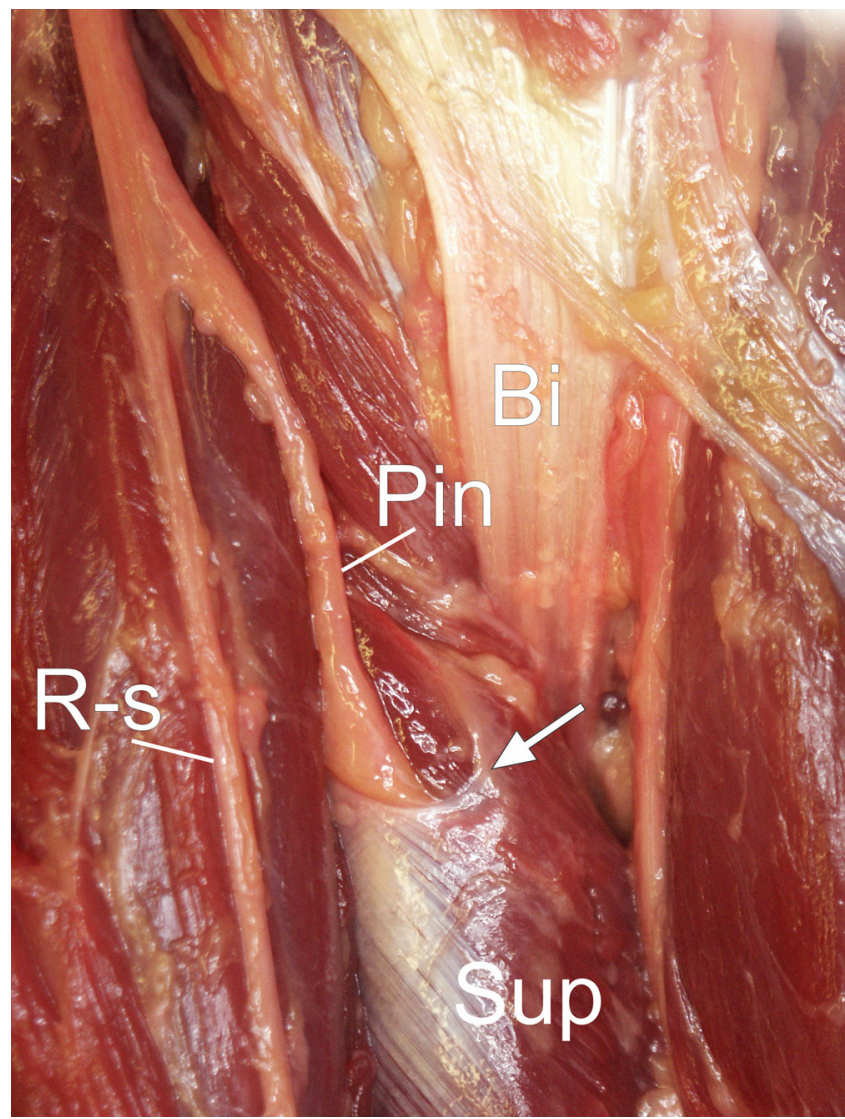

Fig. 28. Anatomical dissection. Branches of the radial nerve. The superficial sensory branch of the radial nerve is seen (R-s). The posterior interosseous nerve (Pin) is seen to course deep to a well developed arcade of Frohse (arrow) as it enters the supinator muscle (Sup). Bi, biceps.

Please cite this article in press as: De Maeseneer M, et al. Ultrasound of the elbow with emphasis on detailed assessment of ligaments, tendons, and nerves. Eur J Radiol (2015), http://dx.doi.org/10.1016/j.ejrad.2014.12.007 


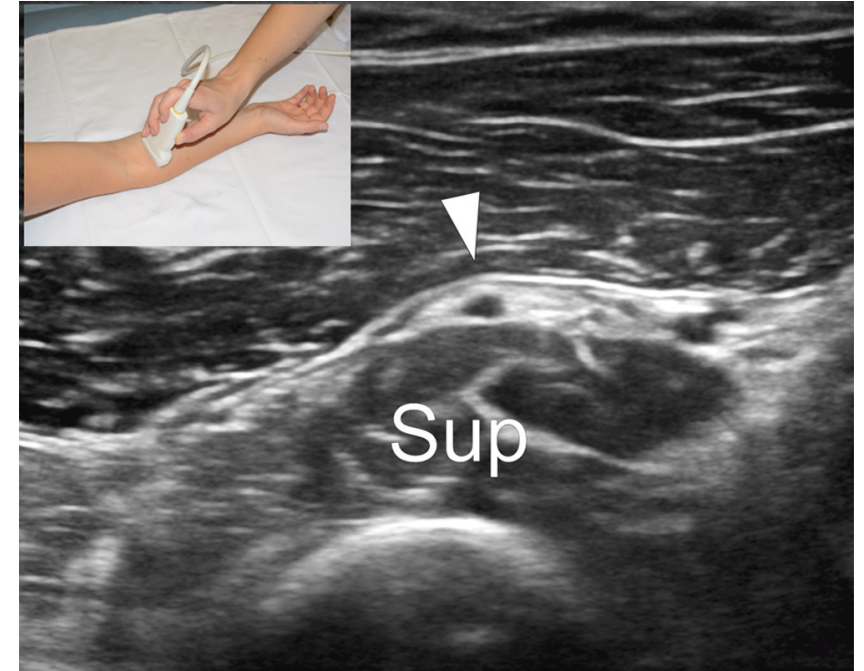

Fig. 29. Transverse ultrasound image. The posterior interosseous nerve is located deep to band like arcade of Frohse (arrowhead) as it enters the supinator muscle (Sup). Insert, probe position.

\subsection{Radial nerve}

Along the posterior aspect of the humerus the radial nerve courses in the spiral groove where it may be injured with humeral fractures. The nerve then has an anterolateral course deep to the brachioradialis muscle. Just before the proximal edge of the supinator muscle the radial nerve divides in the superficial radial nerve and the posterior interosseous nerve (Fig. 28) [11]. The superficial radial nerve is a sensory nerve and continuous distally to emerge adjacent to the tendons of the first extensor compartment where it can be easily discerned. In this area Wartenberg syndrome, compression of the radial nerve may occur. The posterior interosseous nerve enters the supinator muscle between its two heads. At the entrance point the nerve is covered by the arcade of Frohse. The arcade of Frohse is a fibrous band; it may be quite well developed in some individuals but nearly absent in others (Figs. 29 and 30). A thick arcade of Frohse is a potential cause of nerve compression. The posterior interosseous nerve is a motor nerve and compression

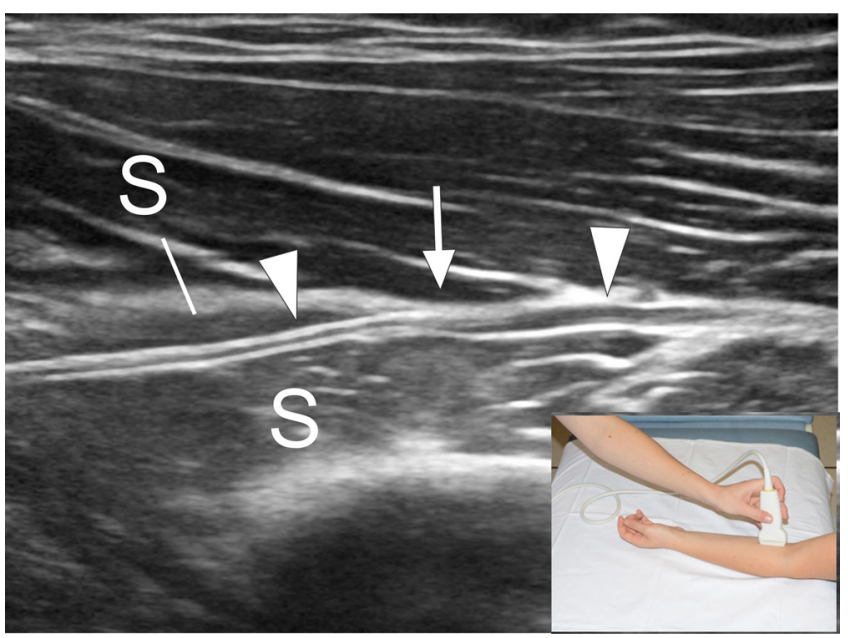

Fig. 30. Longitudinal image of posterior interosseous nerve. Posterior interosseous nerve (arrowheads) is seen as it courses between superficial and deep heads of the supinator muscle (S). Note slight caliber change of the nerve as it enters the supinator muscle (arrow). Insert, probe position.

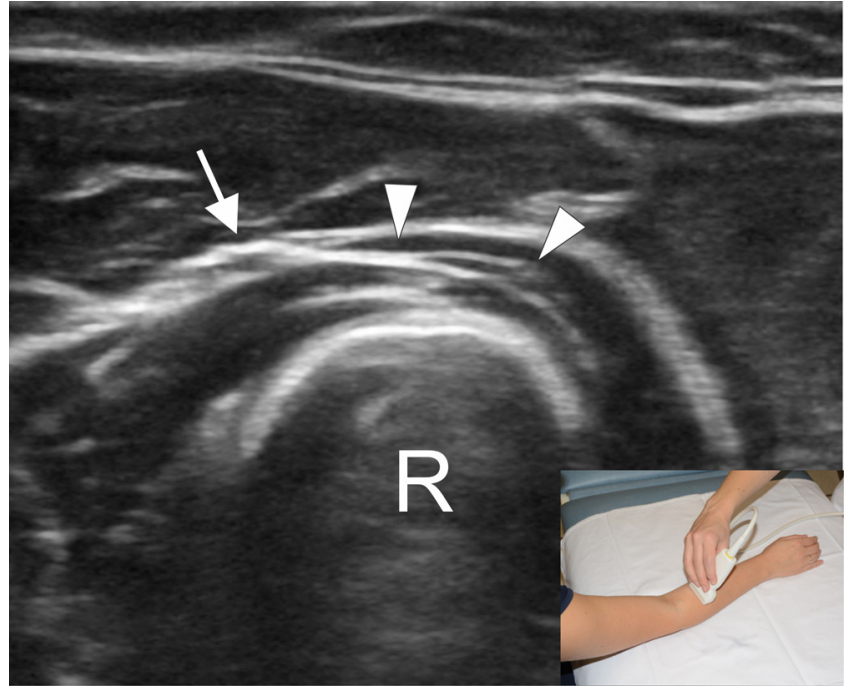

Fig. 31. Transverse ultrasound image with arm in pronation. Linear fatty plane between heads of supinator muscle (arrowheads) corresponds to course of posterior interosseous nerve. The exit point of the nerve from the muscle can be seen (arrow). R, radius. Insert, probe position.

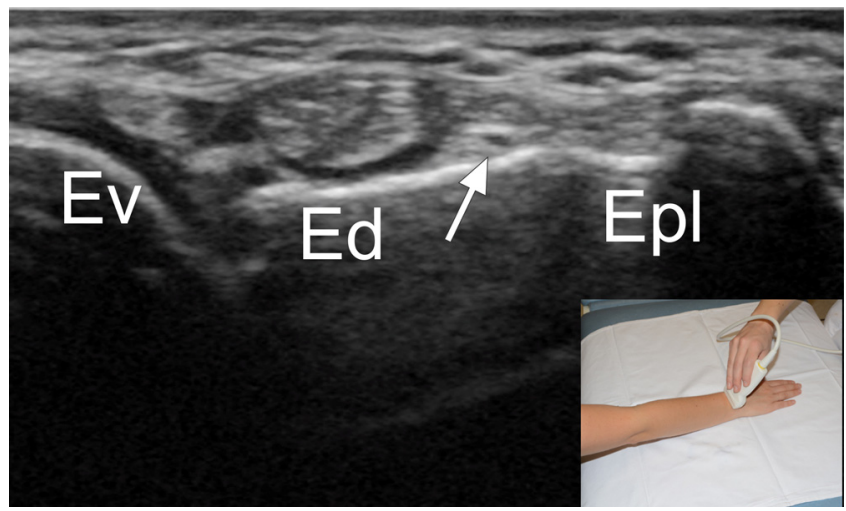

Fig. 32. At the level of the distal radius the posterior interosseous nerve (arrow) can be seen deep in between the extensor digitorum (Ed) and extensor pollicis longus (Epl) tendons. Ev, extensor digiti minimi.

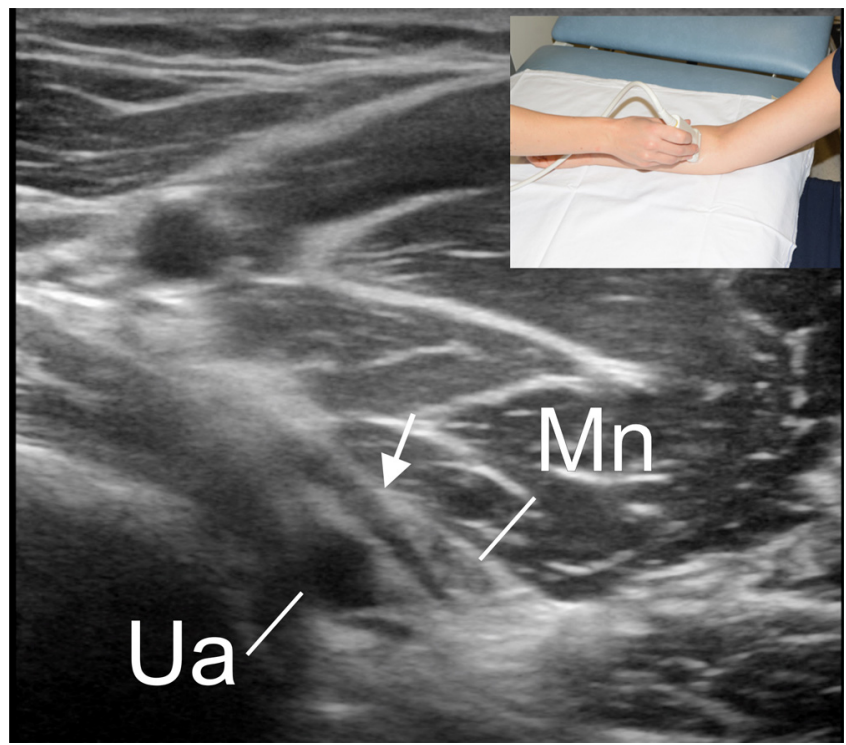

Fig. 33. Transverse ultrasound. Median nerve is seen superficial to the short head of pronator muscle (arrow). This head can typically be seen at the level where the ulnar artery (Ua) courses just deep to the median nerve.

Please cite this article in press as: De Maeseneer M, et al. Ultrasound of the elbow with emphasis on detailed assessment of ligaments, tendons, and nerves. Eur J Radiol (2015), http://dx.doi.org/10.1016/j.ejrad.2014.12.007 


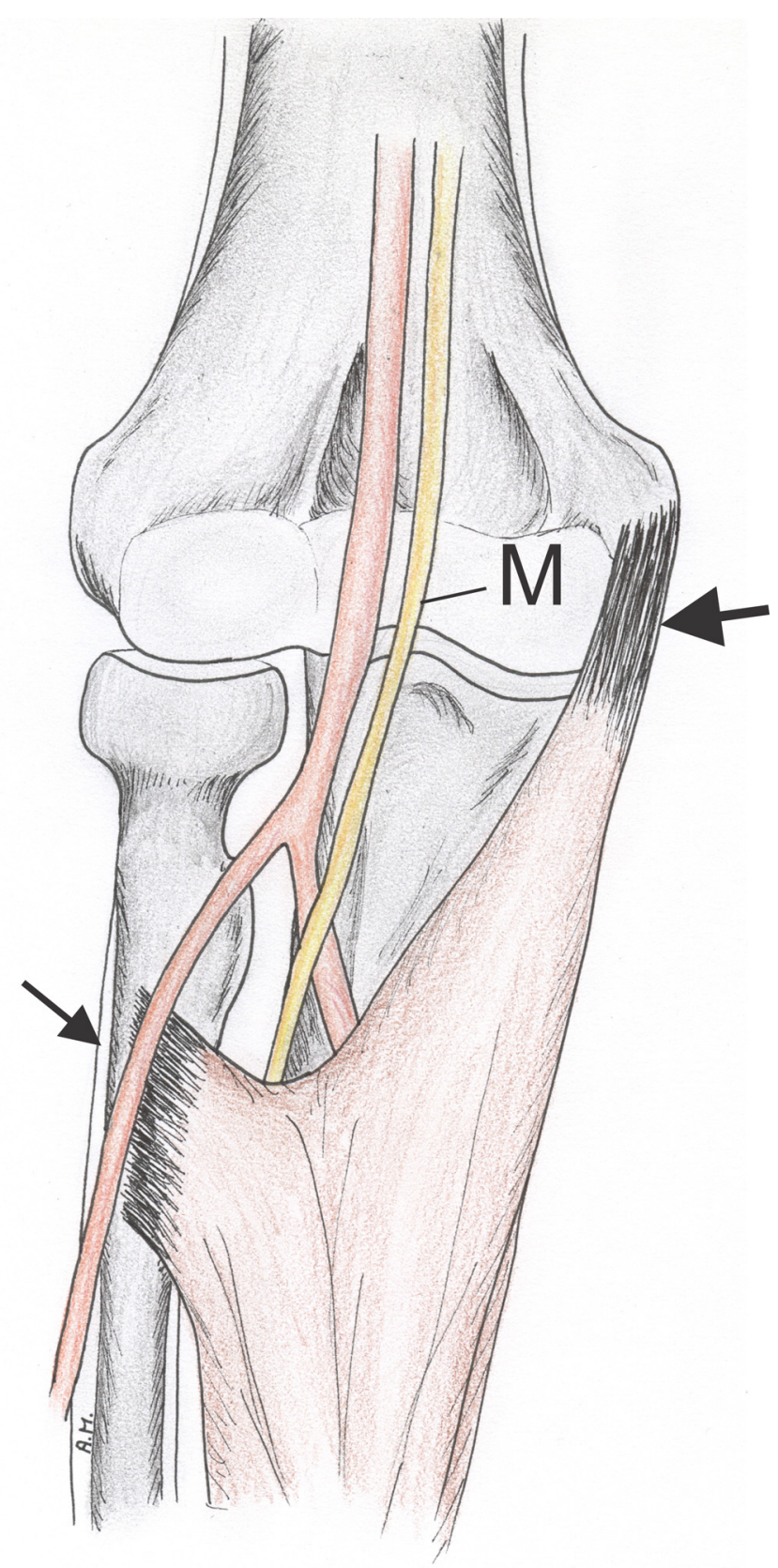

Fig. 34. Drawing of flexor digitorum superficialis muscle. Radial and humeral heads (arrows) of this muscle are seen to form a bridge underneath which the median nerve (M, yellow) courses.

causes the syndrome of finger drop, which should be differentiated from wrist drop that occurs with more proximal lesions of the radial nerve [11-14]. Despite the fact that this branch is a motor nerve it also may cause a pain syndrome that may be difficult to differentiate from pain that occurs with tennis elbow. As a rule of thumb the posterior interosseous nerve should be examined when findings at the level of the extensor tendons are inconclusive. An important sign of neuropathy of the PIN is nerve thickening. Unfortunately no standard values have been described. In addition, it is normal for the nerve to be slightly thicker before it enters the supinator muscle, as it gives off delicate muscle branches once it enters this muscle. This can be well appreciated in the sagittal plane. A significant advantage of ultrasound is also that a comparison with the asymptomatic side can be made.

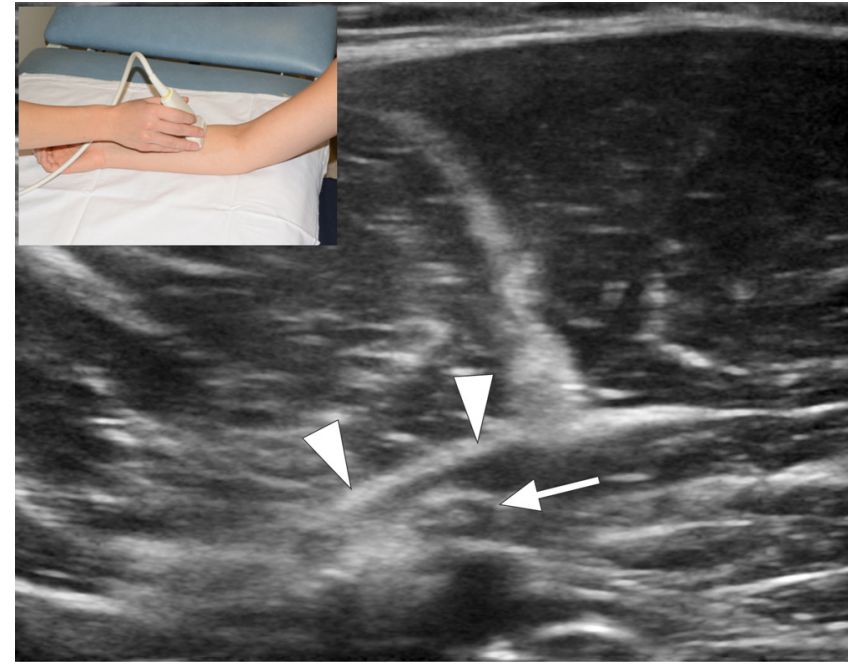

Fig. 35. Transverse ultrasound. Connection between two heads of flexor digitorum superficialis (arrowheads) referred to as sublimis bridge is seen. Median nerve (arrow) courses deep to it. Insert, probe position.

The exit point of the PIN from the supinator muscle is located posteriorly and is also an area that should be investigated (Fig. 31). This can be done by bringing the forearm in pronation so that the course through the muscle and the exit point is brought in view. The PIN can be followed distally throughout the forearm and to level of the distal aspect of the radius, where it can be seen deep to the 4 th extensor compartment (Fig. 32).

\subsection{Median nerve}

At the level of the elbow fold the median nerve can be easily identified by the 'BAM' relationship which we discussed previously. From lateral to medial we observe the biceps tendon, brachial artery, and median nerve (Fig. 17). There are many potential compression areas of the median nerve which include the ligament

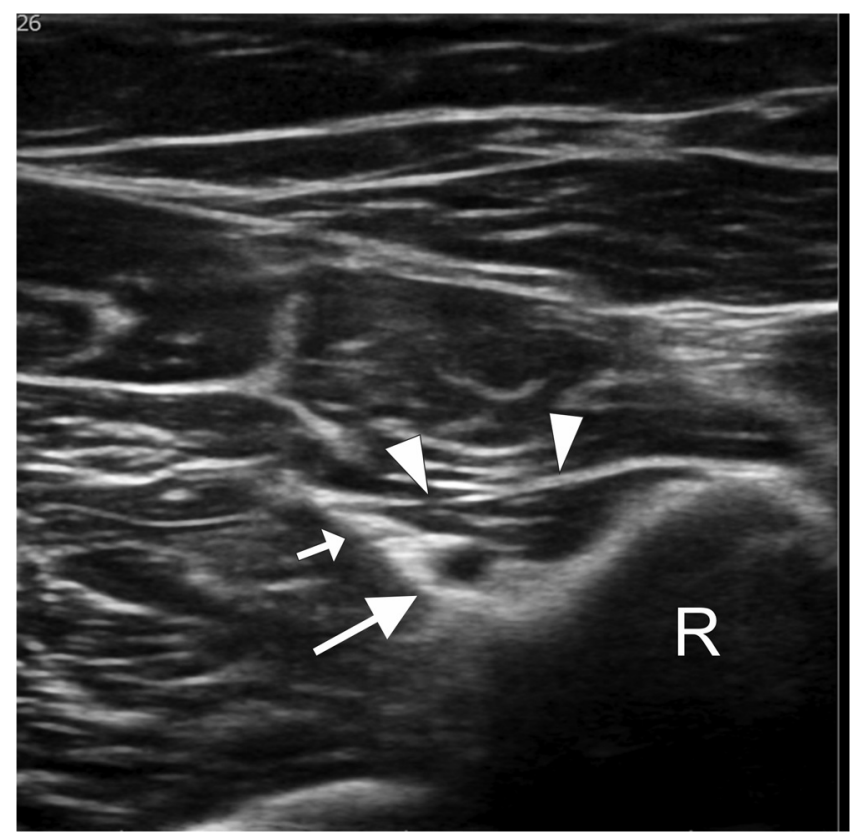

Fig. 36. Transverse ultrasound image at origin of flexor pollicis muscle (arrows) Note triangular shape. It can be readily identified by asking the patient to move the thumb. Anterior interosseous nerve (short arrow) and artery (longer arrow) course alongside the edge of this muscle. $R$, radius. 


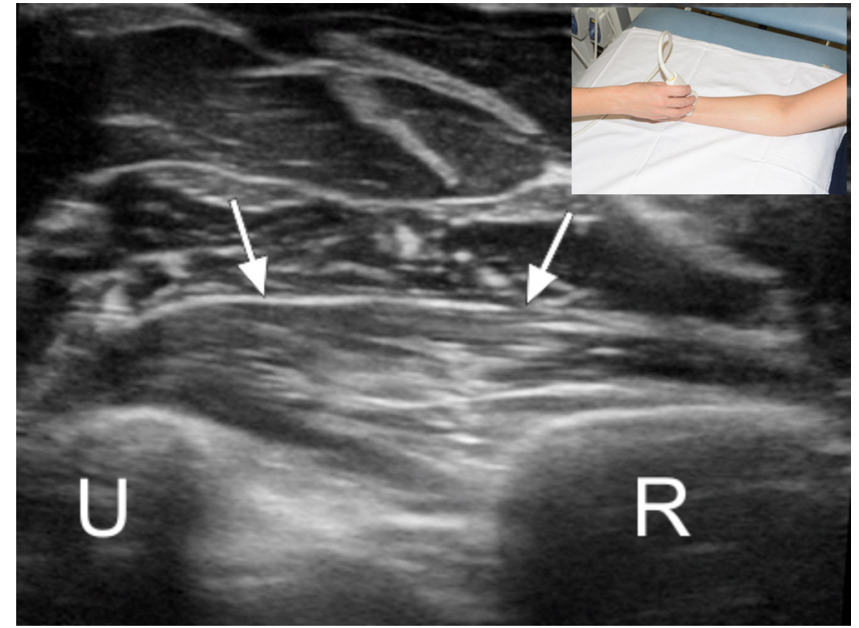

Fig. 37. Transverse ultrasound image at level of pronator quadratus muscle (arrows) in distal forearm. Atrophy and fatty infiltration of this muscle may occur with anterior interosseous nerve involvement. $\mathrm{U}$, ulna; $\mathrm{R}$, radius. Insert, probe position.

of Struthers, lacertus fibrosus, between the pronator heads, the sublimis bridge, and anterior interosseous nerve compression syndrome [11-13]. The ligament of Struthers consists of a fibrous band that courses from a supracondylar process of the humerus to the medial epicondyle. The lacertus fibrosis has been previously described in the paragraph about the biceps tendon. The size and thickness of the lacertus fibrosis is variable however. It can be quite thin and in that case it would be unlikely for it to cause any compression.

The median nerve can be compressed between the large humeral and small ulnar head of the pronator teres muscle (Fig. 33). The method to identify this compression area is to follow the median nerve distally from the elbow fold. The ulnar artery splits of from the brachial artery and courses medially. When the ulnar artery becomes positioned precisely underneath the median nerve a band-like structure is seen between the median nerve and the ulnar artery. This band like structure corresponds to the short head of the pronator teres muscle. The short head of the pronator teres is variable in size. It may be band-like, a thick muscle belly, or absent.

The next compression area of the median nerve is located more distally and corresponds to the sublimis bridge. The sublimis bridge is a fibrous arch between the radial and humeral heads of the flexor digitorum superficialis muscle (Figs. 34 and 35). As the name indicates, if forms an easily recognizable bridge over the median nerve slightly distal to the pronator teres heads.

The anterior interosseous branch of the median nerve splits off from the median nerve. Injury of this nerve causes Kiloh Nevin syndrome with an inability for the patient to pinch between the thumb and 2nd finger. It is a thin branch that may be more difficult to visualize. A good trick, however, is to displace the probe distally until the triangular origin of the flexor muscle for the thumb is brought in view (Fig. 36). Correct identification of this muscle can be confirmed by asking the patient to make movements with his thumb. The anterior interosseous nerve will course just adjacent to the ulnar border of this muscle origin. More distally, the nerve may be seen adjacent to the artery and anterior to the interosseous membrane. A helpful indirect finding indicating neuropathy of the anterior interosseous nerve is fatty atrophy of the pronator quadratus muscle (Fig. 37) [15].

\section{Conclusion}

Ultrasound is an excellent imaging modality for assessment of ligaments, tendons, and nerves about the elbow. Advantages of ultrasound are its high resolution, its dynamic capability and the ability to compare findings with the asymptomatic side.

In this review, we provide valuable tips and tricks that can be used to depict elbow anatomy. Although the technique is operator dependent and experience is necessary, these tricks may significantly shorten learning curves. For better comprehension of anatomic features of different areas, we included anatomical dissections.

\section{Conflict of interest}

The authors have no conflict of interest.

\section{Acknowledgments}

We thank Jan Gielen, MD, PhD, Antwerp, Belgium and Tjeerd Jager, MD, Aalsters Stedelijk Ziekenhuis, Aalst, Belgium. We acknowledge Aloka-Hitachi, Belgium for providing an F75 demo ultrasound system.

\section{References}

[1] Konin GP, Nazarian LN, Walz DM. US of the elbow: indications, technique, normal anatomy, and pathologic conditions. Radiographics 2013;33(July-August (4)):E125-47.

[2] Husarik DB, Saupe N, Pfirrmann CW, Jost B, Hodler J, Zanetti M. Ligaments and plicae of the elbow: normal MR imaging variability in 60 asymptomatic subjects. Radiology 2010;257(October (1)):185-94.

[3] Sanal HT, Chen L, Haghighi P, Trudell DJ, Resnick DL. Annular ligament of the elbow: MR arthrography appearance with anatomic and histologic correlation. AJR Am J Roentgenol 2009;193(August (2)):W122-6.

[4] Stewart B, Harish S, Oomen G, Wainman B, Popowich T, Moro JK. Sonography of the lateral ulnar collateral ligament of the elbow: study of cadavers and healthy volunteers. AJR Am J Roentgenol 2009;193(December (6)):1615-9.

[5] Teixeira PA, Omoumi P, Trudell DJ, Ward SR, Lecocq S, Blum A, Resnick DL. Ultrasound assessment of the lateral collateral ligamentous complex of the elbow: imaging aspects in cadavers and normal volunteers. Eur Radiol 2011;21(July (7)):1492-8

[6] Hayter CL, Adler RS. Injuries of the elbow and the current treatment of tendon disease. AJR Am J Roentgenol 2012;199(September (3)):546-57.

[7] Buck FM, Zoner CS, Cardoso F, Gheno R, Nico MA, Trudell DJ, Randall TD, Resnick D. Can osseous landmarks in the distal medial humerus be used to identify the attachment sites of ligaments and tendons: paleopathologic-anatomic imaging study in cadavers. Skeletal Radiol 2010;39(September (9)):905-13.

[8] Zoner CS, Buck FM, Cardoso FN, Gheno R, Trudell DJ, Randall TD, Resnick D. Detailed MRI-anatomic study of the lateral epicondyle of the elbow and its tendinous and ligamentous attachments in cadavers. AJR Am J Roentgenol 2010;195(September (3)):629-36.

[9] Brigido MK, De Maeseneer M, Morag Y. Distal biceps brachii. Semin Musculoskelet Radiol 2013;17(February (1)):20-7.

[10] Gheno R, Zoner CS, Buck FM, Nico MA, Haghighi P, Trudell DJ, Resnick D. Accessory head of biceps brachii muscle: anatomy, histology, and MRI in cadavers. AJR Am J Roentgenol 2010;194(January (1)):W80-3.

[11] Miller TT, Reinus WR. Nerve entrapment syndromes of the elbow, forearm, and wrist. AJR Am J Roentgenol 2010;195(September (3)):585-94.

[12] Husarik DB, Saupe N, Pfirrmann CW, Jost B, Hodler J, Zanetti M. Elbow nerves: MR findings in 60 asymptomatic subjects - normal anatomy, variants, and pitfalls. Radiology 2009;252(July (1)):148-56.

[13] Martinoli C, Bianchi S, Gandolfo N, Valle M, Simonetti S, Derchi LE. US of nerve entrapments in osteofibrous tunnels of the upper and lower limbs. Radiographics 2000; October [Spec No: S199-213; discussion S213-7. Erratum in: Radiographics 2000;20(November-December(6)):1818].

[14] Ferdinand BD, Rosenberg ZS, Schweitzer ME, Stuchin SA, Jazrawi LM, Lenzo SR, Meislin RJ, Kiprovski K. MR imaging features of radial tunnel syndrome: initial experience. Radiology 2006;240(July (1)):161-8.

[15] Gyftopoulos S, Rosenberg ZS, Petchprapa C, Increased MR. signal intensity in the pronator quadratus muscle: does it always indicate anterior interosseous neuropathy? AJR Am J Roentgenol 2010;194(February (2)):490-3. 